submitted to the Physical Review B

\title{
Orbital magnetism in the half-metallic Heusler alloys
}

\author{
I. Galanakis* \\ Institut für Festkörperforschung, Forschungszentrum Jülich, D-52425 Jülich, Germany
}

(Dated: August 27, 2018)

\begin{abstract}
Using the fully-relativistic screened Korringa-Kohn-Rostoker method I study the orbital magnetism in the half-metallic Heusler alloys. Orbital moments are almost completely quenched and they are negligible with respect to the spin moments. The change in the atomic-resolved orbital moments can be easily explained in terms of the spin-orbit strength and hybridization effects. Finally I discuss the orbital and spin moments derived from X-ray magnetic circular dichroism experiments.

PACS numbers: 71.20.Be, 71.20.Lp, 75.50.Cc
\end{abstract}

Introduction. Half-metallic ferromagnets consist a new class of materials which attracted a lot of attention due to their possible applications in spintronics. $\frac{1}{1}$ In these materials the two spin bands have a completely different behavior. While the majority spin band (referred also as spin-up band) shows the typical metallic behavior, the minority spin band (spin-down band) is semiconducting. The spinpolarization at the Fermi level is $100 \%$ and these compounds could maximize the efficiency of the magnetoelectronic devices ${ }^{2}$

de Groot and collaborators were the first to predict the existence of half-metallicity. They calculated the electronic structure of the Heusler alloy NiMnSb and showed that the Fermi level in the minority band falls within a gap while the majority band was metallic 3 Since then a lot of materials have been predicted to be half-metals: other half-Heusler alloys (e.g PtMnSb) $, 4,5$ a large number of the full-Heusler alloys (e.g $\left.\mathrm{Co}_{2} \mathrm{MnGe}\right), 6.7$ the quaternary Heusler alloys, ${ }^{8.9}$ some oxides $\left(\right.$ e.g $\mathrm{CrO}_{2}$ and $\left.\mathrm{Fe}_{3} \mathrm{O}_{4}\right) \stackrel{10}{10}$ the manganites $\left(e . g \mathrm{La}_{0.7} \mathrm{Sr}_{0.3} \mathrm{MnO}_{3}\right)$, the double perovskites $\left(\right.$ e.g. $\left.\mathrm{Sr}_{2} \mathrm{FeReO}_{6}\right), 11$ the pyrites (e.g $\left.\mathrm{CoS}_{2}\right), 12$ the transition metal chalcogenides (e.g CrAs) and pnictides (e.g CrSe) in the zinc-blende or wurtzite structures, 13.14 .15 the europium chalcogenides (e.g EuS $)^{16}$ and the diluted magnetic semiconductors (e.g Mn impurities in Si or GaAs) $\stackrel{17,18}{\Perp}$ Heusler alloys are particularly interesting among these materials due to their very high Curie temperature, which can attend $1000 \mathrm{~K}$ in the case of $\mathrm{Co}_{2} \mathrm{MnSn}$, and the similarity between their crystal structure and the zinc-blende structure adopted by the III-VI and IV-V binary semiconductors like GaAs or $\mathrm{ZnS}{ }^{19}$

Several papers have been devoted to the calculation of the electronic structure of the half-metallic Heusler alloys. All these studies produced a similar description of their magnetic properties, ${ }^{7,20,21}$ In 2002 Galanakis et al. have shown that the appearance of the gaps in these alloys is directly connected to the magnetic spin moments and moreover that the total spin magnetic moment $M_{t}$ scales linearly with the total number of valence electrons $Z_{t}$ following the low $M_{t}=Z_{t}-18$ for the half-Heusler alloys like NiMnSb and $M_{t}=Z_{t}-24$ for the full Heusler alloys like $\mathrm{Co}_{2} \mathrm{MnGe}{ }^{4.6}$ The orbital magnetic moments of these alloys on the other hand have attracted much less attention and results are scarce. Also experimen- tally only in few cases the orbital magnetic moments have been determined via the X-ray magnetic circular dichroic (XMCD) spectra of thin films. ${ }^{22,23}$ In this contribution I will present a study of the atomic-resolved orbital magnetic moments of several Heusler alloys using first-principles calculations.

Calculations Details. To calculate the orbital and spin magnetic moments I used the fully relativistic (FR) version of the Korringa-Kohn-Rostoker (KKR) multiplescattering Green function method where the Dirac equation for the cell-centered potentials in the atomic spheres (ASA) is solved ${ }^{24}$ The Vosko, Wilk and Nusair parameterization $^{25}$ of the local density approximation (LDA) is used for the exchange and correlation potential. This method has been already employed to calculate the effect of the spin-orbit coupling on the minority band gap in the case of half-metallic ferromagnets. ${ }^{26}$ In the case of $\mathrm{NiMnSb}$ and similar half-Heusler alloys it was shown that the spin-orbit induces states within the gap but the effect is very weak and the alloys show a region of very high spinpolarization ( $\sim 99 \%)$ instead of a gap; defects have a much more pronounced effect on the destruction of the $\operatorname{gap} 27$

If I compare the results obtained in this contribution by using the FR-KKR-ASA with the results obtained in Refs. 4 and 6 using the full-potential (FP) KKR method where the scalar-relativistic approximation is employed (the spin-orbit coupling is not taken into account), both versions of the KKR method reproduce a similar description of the spin magnetic moments; the differences are restricted to small deviations in the absolute values of the spin magnetic moments. Both $C 1_{b}$ and $L 2_{1}$ structures of the half- and full-Heusler alloys, respectively, are closepacked structures and ASA is expected to give a good description of their electronic structure with respect to FP. Moreover spin-orbit is a weak effect and only marginally changes the spin moments. I should also note that LDA is known to underestimate the orbital moments by as much as $50 \%$ but reproduces the correct trends. ${ }^{28.29}$

Half-Heusler alloys containing $M n-S b$. The first family I will study is the MnSb-based half-Heusler alloys and in Table I have gathered their magnetic moments. To this family belong the $\mathrm{Fe}-, \mathrm{Co}-\mathrm{Ni}-$ and $\mathrm{PtMnSb}$ which are half-metallic (HM). RhMnSb and IrMnSb are isoelectronic to CoMnSb but the Fermi level falls within 
TABLE I: Spin $\left(m_{\text {spin }}\right)$ and orbital $\left(m_{\text {orbit }}\right)$ magnetic moments in $\mu_{B}$ for the XMnSb half-Heusler compounds. The last three columns are the total spin and orbital magnetic moment and their sum, respectively

\begin{tabular}{|c|c|c|c|c|c|c|c|c|c|}
\hline \multicolumn{10}{|c|}{ MnSb-based half-Heusler alloys } \\
\hline & $m_{\mathrm{spin}}^{X}$ & $m_{\text {orbit }}^{X}$ & $m_{\mathrm{spin}}^{M n}$ & $m_{\text {orbit }}^{M n}$ & $m_{\mathrm{spin}}^{S b}$ & $m_{\text {orbit }}^{S b}$ & $m_{\text {spin }}^{\text {total }}$ & $m_{\text {orbit }}^{\text {total }}$ & $m^{\text {total }}$ \\
\hline FeMnSb & -0.973 & -0.060 & 2.943 & 0.034 & -0.040 & -0.002 & 1.958 & -0.028 & 1.930 \\
\hline $\mathrm{CoMnSb}$ & -0.159 & -0.041 & 3.201 & 0.032 & -0.101 & -0.001 & 2.959 & -0.010 & 2.949 \\
\hline $\mathrm{NiMnSb}$ & 0.245 & 0.015 & 3.720 & 0.027 & -0.071 & -0.001 & 3.951 & 0.040 & 3.991 \\
\hline $\mathrm{CuMnSb}$ & 0.132 & 0.006 & 4.106 & 0.032 & 0.028 & -0.006 & 4.335 & 0.032 & 4.367 \\
\hline RhMnSb & -0.136 & -0.033 & 3.627 & 0.035 & -0.141 & -0 & 3.360 & 0.001 & 3.361 \\
\hline PdMnSb & 0.067 & 0.007 & 4.036 & 0.028 & -0.117 & -0 & 4.027 & 0.035 & 4.062 \\
\hline $\mathrm{AgMnSb}$ & 0.106 & 0.006 & 4.334 & 0.031 & 0.040 & -0.007 & 4.556 & 0.029 & 4.585 \\
\hline $\mathrm{IrMnSb}$ & -0.201 & -0.094 & 3.431 & 0.092 & -0.109 & -0.001 & 3.130 & -0.004 & 3.126 \\
\hline PtMnSb & 0.066 & 0.006 & 3.911 & 0.057 & -0.086 & 0 & 3.934 & 0.063 & 3.997 \\
\hline $\mathrm{AuMnSb}$ & 0.134 & 0.021 & 4.335 & 0.027 & 0.056 & -0.006 & 4.606 & 0.044 & 4.650 \\
\hline
\end{tabular}

the minority valence band and the HM is lost (the total spin moments are slightly above the ideal value of $\left.3 \mu_{B}\right)$. The Cu-, Ag- and AuMnSb have 23 valence electrons and if they were HM they should have a total spin moment of $5 \mu_{B}$, but as it was shown in Ref. 4 this value is practically impossible to get; it is energetically more favorable to loose the HM. As a result also the spin moments of the $\mathrm{Sb}$ atoms are now parallel to the spin moments of the Mn atoms contrary to the other compounds.

The orbital moments are small with respect to the spin moments and only in the case of IrMnSb the $m_{\text {orbit }}^{I r}$ approaches the $0.1 \mu_{B}$. In the case of the Sb atoms, the $s p$ bands lay low in energy and are almost completely filled for both spin directions ${ }^{4}$ There is a only a very small majority spin $p$-weight around the Fermi level due to the antibonding $p$ - $d$ hybrids. As a result the antimonium orbital moment is practically zero for all compounds.

Mn atoms posses a large spin-magnetic moment in all Heusler alloys. The Mn spin-up states are practically completely occupied while Mn admixture in the occupied minority $d$ states is limited; it is mainly the $\mathrm{X}$ atom which dominates the minority bonding $d$ states .4 Mn orbital moment is less than $0.1 \mu_{B}$ is in all cases and remains parallel to the spin moment following the 3rd Hund rule. The latter rule, although derived for atoms, stands also for solids with few exceptions ${ }^{30}$ It states that if the $d$ band is more than half-filled (Mn has $7 d$-electrons) then the spin and orbital moments should be parallel. Increasing the valence of the $\mathrm{X}$ atom by one electron either following the $3 d$ series $(\mathrm{Fe} \rightarrow \mathrm{Co} \rightarrow \mathrm{Ni} \rightarrow \mathrm{Cu})$ or the $4 d$ series $(\mathrm{Rh} \rightarrow \mathrm{Pd} \rightarrow \mathrm{Ag})$ only scarcely changes the Mn orbital moment while there are significant variations in the value of the $\mathrm{Mn}$ spin moment. If now the $\mathrm{X}$-atom changes along the $5 d$-elements series $(\mathrm{Ir} \rightarrow \mathrm{Pt} \rightarrow \mathrm{Au})$, the increase of the Mn spin moment by $\sim 0.5 \mu_{B}$ at every step is accompanied by a large decrease of the Mn orbital moment which is practically halved. The increase of the spin moment is expected since the hybridization between $\mathrm{Mn}$ and a $d$ atom decreases as the valence of the $d$ atom increases leading to a more atomiclike electronic structure around the Mn site. The large effect on the Mn orbital moment in the case of the $5 d$ atoms has been already discussed in Ref. 30, where using perturbation theory it was shown that the large spin-orbit coupling of the $5 d$ elements has a large effect on the orbital moment of the $3 d$ neighboring atoms in the case of alloys.

Finally for the $\mathrm{X}$ atom the orbital moment follows the Hund's rules and is always parallel to the spin magnetic moment. Note that the Fe, Co, Rh and Ir spin moments are antiparallel with respect to the $\mathrm{Mn}$ atom. The orbital moment follows the changes of the spin moment and it increases as the number of valence electrons increase. As I substitute Co for $\mathrm{Fe}$ the orbital moment increases from $-0.06 \mu_{B}$ to $-0.04 \mu_{B}$ and then to $0.015 \mu_{B}$ for $\mathrm{Ni}$ in NiMnSb. The absolute value of the orbital moment depends strongly also on the spin-orbit coupling. This is clearly seen if I compare Ir with Co. Both atoms have similar spin moments; $-0.16 \mu_{B}$ for Co and $-0.20 \mu_{B}$ for Ir. On the other hand cobalt's orbital moment is $-0.04 \mu_{B}$ while the Ir orbital moment is double as much $\left(-0.09 \mu_{B}\right)$. Also hybridization plays an important role on the value of the orbital moment, e.g in FePt Fe has a spin moment of $2.9 \mu_{B}$ instead of $-1.0 \mu_{B}$ in FeMnSb but the Fe orbital moment is similar in both cases; its absolute value is 0.07 $\mu_{B}$ for FePt and $0.06 \mu_{B}$ for FeMnSb ${ }^{31}$

Orbital moments from first-principle calculations exist for the Ni-, Pd- and PtMnSb compounds obtained using the full-potential linear muffin-tin orbitals method (FPLMTO) ${ }^{32}$ While results for $\mathrm{NiMnSb}$ are similar to the present calculations this is not the case for the $\mathrm{Pd}$ and $\mathrm{Pt}$ atoms in $\mathrm{PdMnSb}$ and $\mathrm{PtMnSb}$ compounds. FPLMTO predicts that their orbital moment is antiparallel to the spin moment contrary to the present calculations. This difference can arise from the treatment of the spin-orbit coupling. Whilst in the present calculations the Dirac equations are solved, in the case of the FPLMTO study the spin-orbit coupling is treated as a perturbation and since orbital moments are very small this can lead to such small deviations.

Finally it was shown in Ref. 33 that the orbital moment is proportional to the difference between the number of states of majority and minority spin at the Fermi level: $m_{\text {orbit }} \propto n^{\uparrow}\left(E_{\mathrm{F}}\right)-n^{\downarrow}\left(E_{\mathrm{F}}\right)$. In the case of the half-metallic systems $n^{\downarrow}\left(E_{\mathrm{F}}\right)=0$ and thus the total orbital moment 
TABLE II: Spin $\left(m_{\text {spin }}\right)$ and orbital $\left(m_{\text {orbit }}\right)$ magnetic moments in $\mu_{B}$ for the $\mathrm{X}_{2} \mathrm{YZ}$ full-Heusler compounds. The last three colums are the total spin and orbital magnetic moments and their sum, respectively

\begin{tabular}{rrrrrrrrr}
\hline \hline & \multicolumn{7}{c}{ Half-ferromagnetic full-Heusler alloys } \\
\hline & $m_{\text {spin }}^{X}$ & $m_{\text {orbit }}^{X}$ & $m_{\text {spin }}^{Y}$ & $m_{\text {orbit }}^{Y}$ & $m_{\text {spin }}^{Z} m_{\text {orbit }}^{Z}$ & $m_{\text {spin }}^{\text {total }}$ & $m_{\text {orbit }}^{\text {total }}$ & $m^{\text {total }}$ \\
\hline $\mathrm{Co}_{2} \mathrm{MnAl}$ & 0.745 & 0.012 & 2.599 & 0.013 & -0.091 & -0 & 3.998 & 0.038 \\
$\mathrm{Co}_{2} \mathrm{MnSi}$ & 0.994 & 0.029 & 3.022 & 0.017 & -0.078 & 0.001 & 4.932 & 0.076 \\
$\mathrm{Co}_{2} \mathrm{MnGe}$ & 0.950 & 0.030 & 3.095 & 0.020 & -0.065 & 0.001 & 4.931 & 0.081 \\
$\mathrm{Co}_{2} \mathrm{MnSn}$ & 0.905 & 0.038 & 3.257 & 0.025 & -0.079 & 0 & 4.988 & 0.101 \\
$\mathrm{Co}_{2} \mathrm{CrAl}$ & 0.702 & 0.012 & 1.644 & 0.008 & -0.082 & 0 & 2.966 & 0.033 \\
$\mathrm{Co}_{2} \mathrm{FeAl}$ & 1.094 & 0.045 & 2.753 & 0.060 & -0.095 & -0 & 4.847 & 0.149 \\
$\mathrm{Fe}_{2} \mathrm{MnAl}$ & -0.311 & -0.015 & 2.633 & 0.014 & -0.016 & 0.001 & 1.994 & -0.014 \\
$\mathrm{Mn}_{2} \mathrm{VAl}$ & -1.398 & -0.034 & 0.785 & -0.009 & 0.013 & 0.005 & -1.998 & -0.073 \\
$\mathrm{Rh}_{2} \mathrm{MnAl}$ & 0.304 & -0.011 & 3.431 & 0.034 & -0.037 & -0.001 & 4.002 & 0.011 \\
\hline \hline
\end{tabular}

should be parallel to the total spin moment. This is not the case always as can be seen in Table In Ref. 33 it was assumed that the $t_{2 g}$ and $e_{g}$ states are degenerate and the local DOS of all atoms is a Lorentzian; thus the applicability of this relation is restricted.

Half-metallic full-Heusler alloys. In the second part of my study I will concentrate on the half-metallic fullHeusler alloys and in Table III I have gathered my results. The orbital moments are quite small like the halfHeuslers. In all cases with the exception of Rh atom in $\mathrm{Rh}_{2} \mathrm{MnAl}$ the Hunds rules are obeyed; note that for $\mathrm{V}$ in $\mathrm{Mn}_{2} \mathrm{VAl}$ the spin and orbital moments are antiparallel since $\mathrm{V} d$ valence shell is less than half-filled. The orbital moments of the $s p$ atoms (Z sites) are almost zero for all cases as in the half-Heuslers

The $\mathrm{Co}_{2} \mathrm{Mn}-\mathrm{Z}$ type compounds are the most interesting since they present the highest Curie temperature among the known half-metals 19 The comparison between the $\mathrm{Al}$ and $\mathrm{Si}$ compounds, which have one valence electrons difference, reveals large changes in their magnetic properties. The Co spin moment increases by nearly 0.25 $\mu_{B}$ and the Co orbital moment follows this change since it is more than double for the Si compound. The increase in the Mn spin moments is proportionally smaller and so do the orbital moments. Substituting now Ge or Sn for $\mathrm{Si}$, which are isovalent systems, has only a weak effect on the spin moments. Co spin moment slightly decreases while the Mn spin moment slightly increases. For both atoms the orbital moments show a small increase with the atomic number.

The next step is to substitute $\mathrm{Cr}$ for $\mathrm{Mn}$ in $\mathrm{Co}_{2} \mathrm{MnAl}$. Co spin moment is not affected by this substitution and so does its orbital moment. Thus the Co orbital moment is mostly induced by the spin-orbit coupling at the Co moment and is insensitive to hybridization with the neighboring sites. Cr moments on the other hand have to account for the missing electron and are considerably smaller than the Mn ones. Substituting Fe for Mn in $\mathrm{Co}_{2} \mathrm{MnAl}$ has a more pronounced effect. Co spin moment increases by $0.35 \mu_{B}$ while its orbital moment is more than tripled. Its also interesting to compare $\mathrm{Co}_{2} \mathrm{FeAl}$ to the isoelectronic $\mathrm{Co}_{2} \mathrm{MnSi}$. Co spin moment in the case of
$\mathrm{Co}_{2} \mathrm{FeAl}$ is slightly larger while the $\mathrm{Co}$ orbital moment is increased by $\sim 50 \%$.

Comparing $\mathrm{Co}_{2} \mathrm{MnAl}$ with $\mathrm{Fe}_{2} \mathrm{MnAl}$ reveals only small changes at the Mn site and the decrease in the total number for valence electrons is taken care by Fe atoms. Substituting now $\mathrm{Rh}$ for $\mathrm{Co}$ in the same compound leads to an increase of both the spin and orbital moments of Mn since the hybridization between $\mathrm{Mn}$ and $\mathrm{Rh} d$ states is considerably smaller than between the Mn and Co $d$ states. Finally I also calculated the properties of $\mathrm{Mn}_{2} \mathrm{VAl}$. The increased hybridization between the Mn and its neighboring $\mathrm{Mn}$ and $\mathrm{V}$ atoms leads to a large orbital moment at the Mn site although its spin moment is halved with respect to the cases above where $\mathrm{Mn}$ occupied the $\mathrm{Y}$ site.

To my knowledge calculations of the orbital moment exist only by Picozzi et al. ${ }^{21}$ for the $\mathrm{Co}_{2} \mathrm{Mn}-\mathrm{Si},-\mathrm{Ge}$ and -Sn compounds. The orbital moment at the Co site was found to be around $0.02 \mu_{B}$ and at the Mn site around $0.008 \mu_{B}$. These moments are slightly smaller than my values. The differences can arise from the treatment of the spin-orbit coupling as perturbation in their calculations.

Experiments. Few experiments dedicated to the orbital magnetism exist on these compounds. These experiments involve the obtaining of the XMCD spectra of thin films. $\mathrm{XMCD}$ is the difference between the absorption spectra for left- and right-circular polarized light involving $2 p$ core states excitations towards unoccupied $d$ states. Elmers and collaborators ${ }^{23}$ derived orbital moments of $0.12 \mu_{B}$ for Co, $0.04 \mu_{B}$ for Cr and $0.33 \mu_{B}$ for Fe in the case of a $\mathrm{Co}_{2} \mathrm{Cr}_{0.6} \mathrm{Fe}_{0.4} \mathrm{Al}$ thin film. If I compare these values with my calculations for the $\mathrm{Co}_{2} \mathrm{CrAl}$ and $\mathrm{Co}_{2} \mathrm{FeAl}$ compounds they are one order of magnitude larger. LDA usually gives orbital moments only halved with respect to experiments ${ }^{29}$ Also the XMCD derived spin moments are half of the theoretical predicted values. On the other hand Kimura et et $a l^{22}$ studied the NiMnSb and PtMnSb films and found that $m_{\text {orbit }}^{\text {total }} / m_{\text {spin }}^{\text {total }}<0.05$ while in my calculations this ratio is around 0.01. The spin moments derived by Kimura et al. experiments are also comparable to the theoretical results. Thus the deviation be- 
tween the present theoretical results and the experiments in Ref. 22 is considerably smaller than when compared to the ones in Ref. 23 .

In both sets of experiments the orbital and spin moments are derived by applying the sum rules to the XMCD spectra. The sum rules have been derived using an ionic model ${ }^{34}$ and their application to itinerant systems, in particular to low symmetry systems, is strongly debated ${ }^{35}$ since XMCD probes mainly the region near the surface of a film. Thus their application to experimental spectra is not straightforward. Elmer's and collaborators sum-rule derived total spin moment is halved not only with respect to the theoretical results but most importantly also with respect to the value derived from the SQUID measurements. This inconsistency even between
XMCD and SQUID measurements on the same sample shows that the application of sum rules to derive the moments in the case of XMCD experiments on films is not really adequate.

Summary. I have studied the orbital magnetism in the half-metallic Half- and Full-Heusler alloys using the Dirac formalism within the framework of the Korringa-KohnRostoker Green's function method. The quenching of the orbital moments is pretty complete and their values are very small with respect to the spin moments. The change in the atomic-resolved orbital moments can be easily explained in terms of the spin-orbit strength and hybridization effects. Moments derived by applying the sum rules to the experimental X-ray dichroic spectra of thin films should be treated with caution.
* Electronic address: I.Galanakis@fz-juelich.de

1 I. Žutić, J. Fabian, and S. Das Sarma, Rev. Mod. Phys. 76, 323 (2004).

2 J. de Boeck, W. van Roy, J. Das, V. Motsnyi, Z. Liu, L. Lagae, H. Boeve, K. Dessein, and G. Borghs, Semicond. Sci. Tech. 17, 342 (2002).

3 R. A. de Groot. F. M. Mueller, P. G. van Engen, and K. H. J. Buschow, Phys. Rev. Lett. 50, 2024 (1983).

${ }^{4}$ I. Galanakis, P. H. Dederichs, and N. Papanikolaou, Phys. Rev. B 66, 134428 (2002).

${ }^{5}$ M. Zhang et al., J. Phys: Condens. Matter 15, 7891 (2003); M. Zhang et al., J. Appl. Phys. 95, 7219 (2003).

6 I. Galanakis, P. H. Dederichs, and N. Papanikolaou, Phys. Rev. B 66, 174429 (2002).

7 S. Fujii, S. Ishida, and S. Asano, J. Phys. Soc. Jpn. 64, 185 (1995); S. Ishida, S. Fujii, S. Kashiwagi, and S. Asano, J. Phys. Soc. Jpn. 64, 2152 (1995).

8 Y. Miura, K. Nagao, and M. Shirai, Phys. Rev B 69, 144413 (2004).

9 I. Galanakis, J. Phys: Condens. Matter 16, 3089 (2004).

10 R. J. Soulen Jr. et al., Science 282, 85 (1998).

11 H. Kato, T. Okuda, Y. Okimoto, Y. Tomioka, K. Oikawa, T. Kamiyama, and Y. Tokura, Phys. Rev. B 69, 184412 (2004).

12 T. Shishidou, A. J. Freeman, and R. Asahi, Phys. Rev. B 64, 180401 (2001).

13 I. Galanakis, Phys. Rev. B 66, 012406 (2002); I. Galanakis and Ph. Mavropoulos, Phys. Rev. B 67, 104417 (2003).

14 S. Sanvito and N. A. Hill, Phys. Rev. B 62, 15553 (2000); B. Sanyal, L. Bergqvist, and O. Eriksson, Phys. Rev. B 68, 054417 (2003); W.-H. Xie, B.-G. Liu, and D. G. Pettifor, Phys. Rev. B 68, 134407 (2003); M. Zhang et al., J. Phys: Condens. Matter 15, 5017 (2003); J.-C. Zheng and J. W. Davenport, Phys. Rev. B 69, 144415 (2004).

15 H. Akinaga, T. Manago, and M. Shirai, Jpn. J. Appl. Phys. 39, L1118 (2000); M. Shirai, J. Appl. Phys. 93, 6844 (2003).

16 M. Horne, P. Strange, W. M. Temmerman, Z. Szotek, A. Svane, and H. Winter, J. Phys.: Condens. Matter 16, 5061 (2004).

17 A. Stroppa, S. Picozzi, A. Continenza, and A. J. Freeman, Phys. Rev. B 68, 155203 (2003).

18 H. Akai, Phys. Rev. Lett. 81, 3002 (1998).
19 P. J. Webster and K. R. A. Ziebeck, in Alloys and Compounds of d-Elements with Main Group Elements. Part 2., edited by H. R. J. Wijn, Landolt-Boörnstein, New Series, Group III, Vol. 19/c (Springer, Berlin), 1988, pp. 75-184.

20 E. Kulatov and I. I. Mazin, J. Phys.: Condens. Matter 2, 343 (1990); S. V. Halilov and E. T. Kulatov, J. Phys.: Condens. Matter 3, 6363 (1991); S. J. Youn and B. I. Min, Phys. Rev. B 51, 10436 (1995); V. N. Antonov, P. M. Oppeneer, A. N. Yaresko, A. Ya. Perlov, and T. Kraft, Phys. Rev. B 56, 13012 (1997).

21 S. Picozzi, A. Continenza, and A. J. Freeman, Phys. Rev. B 66, 094421 (2002).

22 A. Kimura, S. Suga, T. Shishidou, S. Imada, T. Muro, S. Y. Park, T. Miyahara, T. Kaneko, and T. Kanomata, Phys. Rev. B 56, 6021 (1997).

23 H. J. Elmers et al., Phys. Rev. B 67, 104412 (2003).

24 N. Papanikolaou, R. Zeller, and P. H. Dederichs, J. Phys.: Condens. Matter 14, 2799 (2002).

25 S. H. Vosko, L. Wilk, and N. Nusair, Can. J. Phys. 58, 1200 (1980).

26 Ph. Mavropoulos, K. Sato, R. Zeller, P. H. Dederichs, V. Popescu, and H. Ebert, Phys. Rev. B 69, 054424 (2004); Ph. Mavropoulos, I. Galanakis, V. Popescu, and P. H.Dederichs, J. Phys.: Condens. Matter, in press.

27 D. Orgassa, H. Fujiwara, T. C. Schulthess, and W. H. Butler, Phys. Rev. B 60, 13237 (1999); S. Picozzi, A. Continenza, and A. J. Freeman, Phys. Rev. B 69, 094423 (2004).

${ }^{28}$ H Ebert, Rep. Prog. Phys. 59, 1665 (1996).

29 W. Grange, I. Galanakis, M. Alouani, M. Maret, J.-P. Kappler, and A. Rogalev, Phys. Rev. B 62, 1157 (2000).

30 I. Galanakis, P. Ravindran, P.M. Oppeneer, L. Nordström, P. James, M. Alouani, H. Dreyssé, and O. Eriksson, Phys. Rev. B 63, 172405 (2001).

31 I. Galanakis, M. Alouani and H. Dreyssé, Phys. Rev. B 62, 6475 (2000).

32 I. Galanakis, S. Ostanin, M. Alouani, H. Dreyssé, and J. M. Wills, Phys. Rev. B 61, 4093 (2000).

${ }^{33}$ H. Ebert, R. Zeller, B. Drittler, and P. H. Dederichs, J. Appl. Phys. 67, 4576 (1990).

34 P. Carra, B. T. Thole, M. Altarelli, and X. Wang, Phys. Rev. Lett. 70, 694 (1993); B. T. Thole, P. Carra, F. Sette, and G. van der Laan, Phys. Rev. Lett. 68, 1943 (1992).

35 C. T. Chen et al., Phys. Rev. Lett. 75, 152 (1995); R. Wu, 
D. Wang, and A. J. Freeman, Phys. Rev. lett. 71, 3581 (1993); R. Wu and A.J. Freeman, ibid 73, 1994 (1994). 